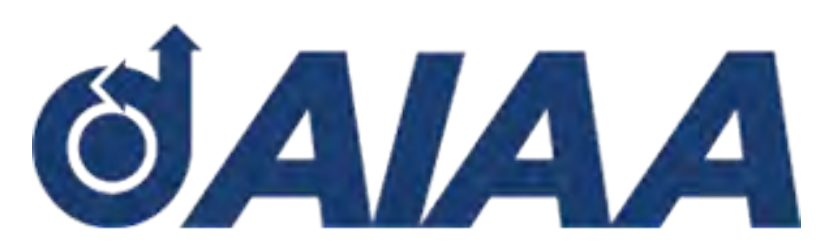

\title{
Analytical Framework for Precise Relative Motion in Low Earth Orbits
}

\begin{tabular}{|c|c|}
\hline J ournal: & J ournal of Guidance, Control, and Dynamics \\
\hline Manuscript ID & 2019-07-G004716.R1 \\
\hline Manuscript Type: & Full Paper \\
\hline $\begin{array}{r}\text { Date Submitted by the } \\
\text { Author: }\end{array}$ & $\mathrm{n} / \mathrm{a}$ \\
\hline Complete List of Authors: & $\begin{array}{l}\text { Galas, Gabriella; Politecnico di Milano, Department of Aerospace Science } \\
\text { and Technology } \\
\text { Colombo, Camilla; Politecnico di Milano, Department of Aerospace } \\
\text { Science and Technology } \\
\text { Lara, Martin }\end{array}$ \\
\hline Subject Index Category: & $\begin{array}{l}31400 \text { Dynamics }<30000 \text { GUIDANCE, CONTROL, AND DYNAMICS } \\
\text { TECHNOLOGY, } 33300 \text { Spacecraft Guidance and Control < } 30000 \\
\text { GUIDANCE, CONTROL, AND DYNAMICS TECHNOLOGY }\end{array}$ \\
\hline \multicolumn{2}{|c|}{$\begin{array}{l}\text { Note: The following files were submitted by the author along with the article. You may review these files } \\
\text { online, if you wish. Acceptance for publication will be based solely on the content of the article. }\end{array}$} \\
\hline 1stReviewSubmission.zip & \\
\hline
\end{tabular}

\section{SCHOLARONE ${ }^{m}$ Manuscripts}




\title{
Analytical Framework for Precise Relative Motion in
}

\section{Low Earth Orbits}

\author{
Gabriella Gaias ${ }^{1}$ and Camilla Colombo ${ }^{2}$ \\ Politecnico di Milano, Milano, 20156, Italy \\ Martin Lara $^{3}$ \\ University of La Rioja, Logroño, 26006, Spain
}

This work presents a practical and efficient analytical framework for the precise modeling of the relative motion in low Earth orbits. Developed to support the design and verification of relative guidance navigation and control algorithms devoted to spacecraft rendezvous for active debris removal applications, only the orbital perturbation due to non-spherically symmetric mass distribution is considered. The relative motion is modeled in mean relative orbital elements, revisiting the available formulations to include the first-order expansion of the effects due to any even zonal harmonics and the second-order expansion of the unperturbed and $J_{2}$ terms. Mean/osculating orbital elements conversions are obtained merging a second-order Hamiltonian approach applied to the $J_{2}$ problem with Kaula's linear perturbation method for the remaining terms of the geopotential. The paper describes the main building blocks of the framework as well as their interfaces, since the key aspect to achieve precision is to set up a fully consistent environment. Results show the achievable accuracy under realistic operational conditions for possible guidance and navigation applications.

\footnotetext{
${ }^{1}$ Research Engineer, Department of Aerospace Science and Technology, Via La Masa 34.

2 Associate Professor, Department of Aerospace Science and Technology, Via La Masa 34.

${ }^{3}$ Researcher, GRUCACI, C/ Madre de Dios 53.
} 


\section{Introduction}

The stringent need of performing active debris removal and satellite life extension, to preserve commercially and scientifically relevant orbits in the low Earth orbit (LEO) belt [1], asks for accurate relative motion models accounting for the perturbation due to non-spherically symmetric mass distribution. The critical targets in this region typically lay on near-circular orbits at an height where the aerodynamic drag is not strong enough to support their natural decay in a reasonable time span [2]. For what concerns noncooperative rendezvous in LEO, the Autonomous Vision Approach Navigation and Target Identification (AVANTI) experiment has demonstrated the feasibility to approach a fully passive object, from approximately $50 \mathrm{~km}$ of separations down to few dozens of meters, relying exclusively on angles-only observations extracted from a monocular, far-range, camera system [3]. At the same time, in addition to formation-flying and rendezvous applications, new concepts of space-based space situational awareness are emerging, to classify the debris population [4]. These foresee spaceborne relative orbit estimation based on angles-only observations, to complement the existing ground-based services. The relative navigation based on angles-only observations is critical due to the weak observability of the relative state from bearing-only information. This impacts the navigation accuracy achievable by onboard systems [5]. And, for ground-in-the-loop applications, the numerical integration of the equation of motions either in Cartesian frame [6] or through the Gauss variational equations (GVEs) [4], is employed. Indeed, a fully analytical formulation able to achieve accuracy performances comparable to numerical schemes, would be beneficial to support the synthesis of spaceborne guidance navigation and control (GNC) algorithms [7].

Several relative motion models have been proposed in the recent years [8]. Among these, orbital elements based approaches are often exploited, following the seminal works [9, 10], which recognized the advantages of linearizing with respect to the elements of the chief satellite. Generally, working in the elements' space allows reducing the linearization errors in the initial conditions, simplifies the inclusion of orbital perturbations, and allows exploiting celestial mechanics methods to identify the most efficient locations of the orbit correction maneuvers when designing relative guidance and control algorithms. The state variables' set can either be constituted by elements' differences between the deputy and chief satellites or functions thereof. In both cases several families can 
be adopted, offering different levels of singularity in their definitions (e.g., classical, non-singular, equinoctial, Hoots [11] elements) or supporting a canonical structure (e.g., Delaunay, Poincarè, Whittaker [12] elements). By focusing only on models including the geopotential perturbation, Gim and Alfriend introduced the geometric method to develop a state transition matrix (STM) including the effect of secular, long-, and short-periodic contributions due to $J_{2}$ to the first order. The core part of this methodology is constituted by the first-order expansion of the secular orbit of the chief satellite, whose state is parametrized in differences of non-singular elements with the argument of true latitude for the satellite anomaly on the orbit. Then the STM is completed by the Jacobian of the mean-to-osculating transformations and by a first-order mapping of the elements' differences into the relative state in a curvilinear co-moving reference frame. This approach has been also developed in equinoctial elements [13]; whereas Sengupta et al. included the second-order Taylor expansion of the unperturbed (i.e., Keplerian) orbit of the chief satellite in the core part [14]. More recently, Yang et al. extended the Gim-Alfriend GA-STM by including the second-order Taylor expansion, while adopting the same parametrization [15]. There, the second-order transition tensor (STT) is developed for both unperturbed and secular solution due to $J_{2}$. Moreover, the mapping of the elements' differences into the relative state also includes the second-order Taylor expansion.

Parallel to these models parametrized in elements' differences, several options employ the so called relative orbital elements (ROEs) inherited from the co-location of geostationary satellites [16] and afterwards adapted to the formation-flying field [17]. By adopting ROEs, Gaias et al. proposed a STM including the effects due to the Earth oblateness for the near-circular case [18]. It is derived by expanding to the first order the mean time derivatives of the chief elements from the Brouwer's satellite theory. Since the expansion is carried out on the derivatives, one obtains the plant matrix corresponding to the linearized relative dynamics, which, by neglecting terms proportional to the chief eccentricity, reduces to a linear time-invariant system. For the general eccentric case, in order to integrate the linearized time-variant system in ROEs, Koenig et al. introduced an intermediate transformation (basically a rotation of the relative eccentricity vector within the orbital plane) to obtain a linear time-invariant system (moreover with plant matrix nilpotent of order 2) equivalent to the original system within the first-order assumption [19]. Note that such transformation can be 
exploited to compute the STM of the relative motion accounting for the first-order secular effects due to any term of the geopotential, as performed in [20], since only even zonal terms give rise to secular effects [21].

The inclusion of the effects produced by the zonal geopotential terms higher than order-2 up to part of $J_{4}$ has been performed exploiting spheroidal orbital elements [22, 23]. In classical elements, instead, Yan et al. extended the core of the GA-STM including the effects due to $J_{2}^{2}, J_{4}$, and $J_{6}$ while the initial osculating elements consistent to a given set of mean elements were obtained by a least squares approach [24]. The majority of the models mentioned so far, also recalled in [8], exploit the geometric method applied to first-order satellite theories. Indeed, the effect on the relative dynamics produced by geopotential terms higher than the oblateness one is tiny and might become significant over longer propagation time spans or large size of the relative orbit. Thus, in presence of errors in the computation of the initial mean elements from a given osculating set using first-order transformations, that contribution is shadowed by the propagation of such initial errors. By considering a second-order Lie-series based approach, at least for the dominant $J_{2}$ term, the overall accuracy obtainable by relative motion models greatly improves [25]. Recent works focus on the extension of the GA-STM computing the mapping, elements' transformation, and core blocks from second-order satellite theories developed through canonical perturbation methods. In particular, in [26] Hoot's satellite theory is extended to second order and the STM is then written in Hoot's variables, whereas Mahajan et al. tackled the full zonal problem [27] as well as the general problem [28] - including secular and periodic effects due to the zonal, sectorial, and tesseral harmonic perturbations - to obtain a first-order STM in equinoctial elements. Compared to the models that include the perturbation effects of the $J_{2}$ harmonic up to first order only, these formulations allow achieving much more accurate propagation results, at the cost of an increased complexity of the functional expressions of the components of the STM.

This work focuses on the set-up of an analytical framework that allows improving the accuracy performances of the simple first-order $J_{2}$-only models, while keeping the minimum level of complexity strictly required. A modular structure is used: the elements' transformations are kept separated from the core propagation module, to exploit the accuracy of the implemented algorithm also using 
available first-order models. In addition, for the elements' conversion, a compact algorithm - suitable also for onboard implementation - is developed. This can include a user-definable order and degree terms of the geopotential, is fully analytical in both transformation directions, and free from singularities (but not applicable in the vicinity of the critical inclination).

The core part of the framework is composed by the STM of the relative motion in mean ROEs, where only the secular effects due to odd zonal harmonics up to order-6 are considered. Here, the use of the differential mean argument of longitude instead of the difference in true argument of latitude (as done in $[10,15]$ ) allows reducing the coupling among the elements of the STM. With respect to the available ROE-based relative motion models, the current analytical formulation generalizes the first-order state transition matrix to include the secular effect of even zonal harmonics without introducing any further assumption (and is valid also for the eccentric case). Moreover, the analytical expression of the second-order state transition tensor accounting for the Keplerian and first-order $J_{2}$ terms is also provided. On need, the approach is easily extendable to include further terms of the expansion.

The second main building block of the framework is the function to perform osculating-to-mean transformation of the absolute orbital elements, needed since GNC algorithms are conveniently developed in the mean ROEs [3, 29-31], whereas the absolute motion of the chief satellite is generally estimated onboard to provide the absolute Cartesian state in an Earth-centered inertial reference frame [32]. The computation of mean elements from the osculating set is based on averaging techniques. The survey in [33] presents an overview of available analytical orbital theories depending on included perturbations and orbit types. The accuracy requirement for formation flying applications is relaxed by the fact that the satellites lie in neighboring orbits and, therefore, part of the conversion-error cancels as soon as one regards the relative mean elements. Moreover, the typical propagation time-scales between orbit corrections rarely exceed few days, especially when the satellites are very close to each other. The most critical requirement for close formation flying is the level of autonomy of the onboard relative GNC system.

Since the oblateness term is the largest coefficient of the geopotential, the periodic corrections due to all zonal, sectorial and tesseral harmonics can be computed combining an approach addressing 
the $J_{2}$ to the second-order with the Kaula first-order method for the remaining higher degree zonal, sectorial and tesseral terms [34, 35]. More recently Spiridonova et al. exploited the same strategy for ground-based LEO orbit monitoring and maintenance, using an iterative method to cancel the short-periodic terms due to $J_{2}$ computed with Eckstein's satellite theory [36, 37]. In this work the conversion function employs a second-order Lie-series based approach, analytically derived up to certain order expansion of the $e J_{2}$ term (where $e$ is the eccentricity of the chief orbit), to cancel the short- and long-periodic terms due to the dominant $J_{2}$ coefficient. Then, the semi-major axis component only is refined through the first-order Kaula method up to the required order and degree term of the geopotential. As the drift in the along-track direction is proportional to the relative semi-major axis, in fact, the highest precision is strictly required only for such component of the orbital elements' set.

The paper is organized as follows: section II describes the framework critically motivating its design choices. Section III focuses on the algorithm to convert from/to mean/osculating elements, providing the numerical comparison with respect to other methodologies in the literature. Section IV addresses the relative motion model in the mean ROE space, explaining the development of the first-order state transition matrix as well as of the second-order state transition tensor. Section $\mathrm{V}$ presents the achievable accuracy results, focusing on both the benefits from the implemented elements' transformation algorithm and the improvements produced by the developed relative motion model. The functional expressions of the transformation algorithms are available in the supplemental appendix to this paper.

\section{Framework definition}

This section describes the framework in terms of actions' flow and use to support the design of relative GNC algorithms. Figure 1 shows two possible configurations, to serve respectively navigation and guidance purposes. For each set-up, gray-background boxes denote information from outside: either inputs to the operations or data required to accomplish the GNC task (e.g., the true observations from sensors $\mathbf{h}(t))$. Outputs, instead, are in dark-background blocks. All operations are implemented on the chief satellite, whose absolute state $\mathbf{y}_{c}$ is known at each current time. In 
noncooperative scenarios, the state of the deputy spacecraft has to be estimated out of the observations collected by the sensors mounted on the chief. Accordingly, in the navigation task, one aims at estimating the relative state at a given time $\delta \overline{\boldsymbol{\alpha}}_{0}$ (parametrized in ROEs, which are defined later in Eq. (1)), so that the residuals between the collected $\mathbf{h}\left(t_{i}\right)$ and modeled $\tilde{\mathbf{h}}\left(t_{i}\right)$ observations of the deputy spacecraft are minimized. Once that the relative state at a certain time is known, the guidance task aims at finding a control policy that brings the relative trajectory to a target one at a later time $\delta \overline{\boldsymbol{\alpha}}^{\mathrm{tar}}$, minimizing the error in the relative state at that time $t$. Examples of implementation of guidance and control schemes are provided in [3, 29-31]. Figure 1 depicts the chain of operations for both navigation and guidance set-ups. At each time $t$, the functions specified over the arrows are computed, following order and direction. The result of each operation is written in the neighboring box.

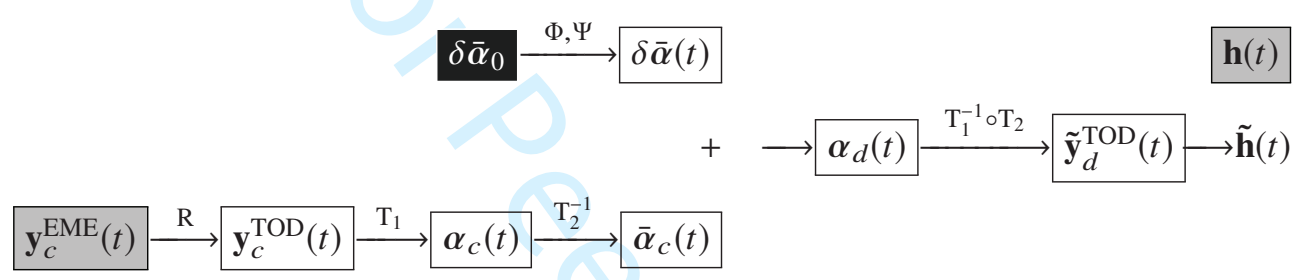

a) Navigation set-up

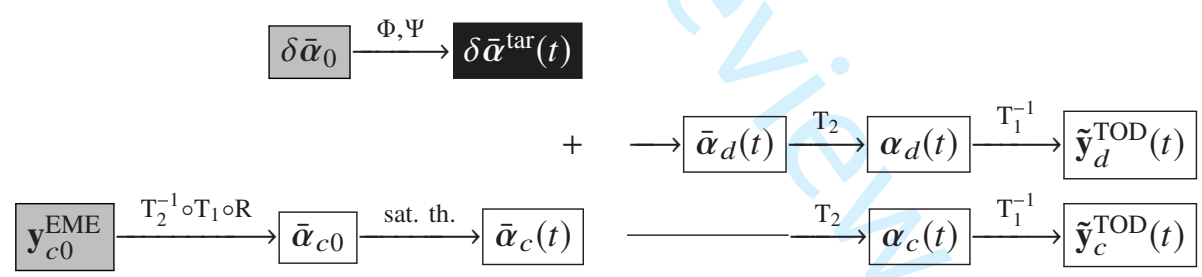

b) Guidance set-up

Fig. 1 Actions' flow of the framework: chain of operations executed at each time $t$.

Regarding the nomenclature adopted in Fig. 1, the absolute orbit of the chief, in LEO usually estimated onboard by processing GNSS position data, is expressed in a Cartesian Earth-centered inertial reference system. A common choice is to employ the Earth Mean Equator and Equinox of J2000 (EME) reference system, given the availability of star catalogs in that frame [38]. The 
conversion between osculating/mean elements, instead, requires the use of the true of date (TOD) reference system, coherently with the arrangement of the geopotential harmonics with respect to the terrestrial equator. Accordingly, the transformation R in Fig. 1 is the rotation from EME to TOD, which is a composition of the precession and nutation rotations [38]. In order to work in the elements' space, the Cartesian absolute state is transformed into the corresponding set of osculating elements through the transformation $\mathrm{T}_{1}$. Here $\boldsymbol{\alpha}=\left(a, u, e_{x}, e_{y}, i, \Omega\right)^{\mathrm{T}}$ is the set of Keplerian non-singular elements, with $e_{x}=e \cos \omega, e_{y}=e \sin \omega$, and $u=\omega+M$ respectively being the $x$ and $y$ components of the eccentricity vector and the spacecraft mean argument of latitude, $a$ the semi-major axis, $\omega$ the argument of the perigee, $M$ the mean anomaly, and $i$ the inclination. The transformation from mean to osculating elements $\mathrm{T}_{2}$ will be explained in details in section III; the ${ }^{\bar{*}}$ notation denotes mean quantities, which therefore are the averaged ones out of $\mathrm{T}_{2}^{-1}$. The relative state in ROEs $\delta \boldsymbol{\alpha}$ is defined as follows:

$$
\begin{aligned}
\delta \boldsymbol{\alpha} & =\left(\delta a, \delta \lambda, \delta e_{x}, \delta e_{y}, \delta i_{x}, \delta i_{y}\right)^{\mathrm{T}} \\
& =\left(\Delta a / a_{c}, \Delta u+\Delta \Omega \cos i_{c}, \Delta e_{x}, \Delta e_{y}, \Delta i, \Delta \Omega \sin i_{c}\right)^{\mathrm{T}}
\end{aligned}
$$

where $\Delta \cdot$ denotes the difference between quantities of the deputy " $\mathrm{d}$ " and chief "c" satellites, $\delta \lambda$ is called the relative mean argument of longitude, and the vectors $\left(\delta e_{x}, \delta e_{y}\right)^{\mathrm{T}}$ and $\left(\delta i_{x}, \delta i_{y}\right)^{\mathrm{T}}$ are respectively known as the relative eccentricity and inclination vectors. As explained in [39], the functional expression of the relative inclination vector in Eq. (1) corresponds to the unit non-coplanar vector between chief and deputy orbits, when $\Delta i$ and $\Delta \Omega$ are small. Whereas the relative eccentricity vector in Eq. (1) is defined as the difference of the eccentricity vectors of deputy and chief satellites, having assumed that the two orbital planes almost coincide (again $\Delta i$ and $\Delta \Omega<<1$ ) so that the projection of $\mathbf{e}_{d}$ on the chief orbital plane is assumed to coincide with the original vector [40]. Note that Han and Yin in [41] remove this last assumption, to obtain a definition that is valid also for eccentric reference orbits (i.e., the additional term that remains after considering small differences of orbital elements is proportional to $e \Delta \Omega$ and thus negligible in LEO for small eccentric orbits). By introducing a polar notation for both relative eccentricity and inclination vectors, the phase angles defined from the axes of nodes of the chief satellite have respectively the meaning of perigee $\varphi$ and ascending node $\theta$ of the relative orbit. And, for almost-bounded relative orbits (i.e., $\delta a \approx 0$ ) the 
minimum distance in the local radial-normal $(\mathrm{RN})$ plane can be related to the difference of $\varphi$ and $\theta$ angles and thus to the phasing of the relative eccentricity/inclination vectors [39], with all the important consequences on spacecraft proximity operations and collision avoidance strategies. Note that the analytical expression of the one-orbit minimum RN distance can be straightaway extended to drifting relative orbits, resulting from non-vanishing relative semi-major axis encountered during a rendezvous or produced by the action of the differential aerodynamic drag, to take into account the shift in the radial direction [42]. Finally the use of $\delta \lambda$, thanks to the term $\Delta \Omega \cos i_{c}$, which is a correction of the basis of the mean argument of latitude for non-coplanar satellites [41], allows decoupling the in-plane and out-of-plane relative motions (in the unperturbed case). This factor is extremely useful when designing impulsive guidance and control algorithms based on the GVEs, fostering the design of reconfiguration relative trajectories directly in the ROE space [43, 44].

By comparing the functional blocks of the framework with the chain of transformations composing the geometric method developed in [10], one can note that, first, in both approaches the core part is constituted by the propagation of the orbital elements based relative state vector in the mean space. Nevertheless, by using $\delta \boldsymbol{\alpha}$ as parametrization, where $\delta \lambda$ is a trivial function of $\Delta u$, simpler expressions of the components of the first-order STM are obtained, because there is no need to relate the expansion of $\dot{u}$ to the difference of true argument of latitude. Second, the mean to osculating transformations $\mathrm{T}_{2}$ are directly employed instead of their Jacobian. In $[10,15]$ the latter is required to subsequently map the differences in osculating elements into the relative state in the local curvilinear co-moving frame. From an engineering point of view, however, it is very convenient to develop guidance and control algorithms directly in the ROE space. Examples are dead-band control strategies on the ROE evolution for formation-keeping $[29,30]$ or delta-v minimum trajectory design through way-points in the ROE space for large reconfigurations [3, 44]. Note that, the additional mapping into the relative state introduces further approximations that may degrade the overall propagation accuracy. For this reason, in comparative studies as [8] the GA-STM method is evaluated splitting its core function from the full formulation. And in angles-only relative navigation cases, where the observability property demands for the best possible accuracy, the observations are modeled as in this framework from $\tilde{\mathbf{y}}_{d}$ and $\mathbf{y}_{c}[4-6]$. Last, considering the structure depicted in 
Fig. 1, the solution of the Kepler's equation is required only by the transformation $\mathrm{T}_{1}^{-1}$, to recover the Cartesian absolute state from the osculating elements set $\boldsymbol{\alpha}$.

The overall accuracy of the framework is assessed by considering each error source introduced in the chain of actions. Errors in the implementation of the block interfaces and the two-way errors in the conversion algorithm $\mathrm{T}_{2}$ introduce artificial sources of error that degrade the overall performance of the relative motion model. The focus of this paper is the precise modeling of the relative motion; thus, in the navigation set-up, the modeled observations $\tilde{\mathbf{h}}$ are computed from $\tilde{\mathbf{y}}_{d}$ and $\mathbf{y}_{c}$ without introducing errors. Moreover, no guidance/control policies are considered and the target relative state at a future time is simply the propagated relative state up to that time. Coherently, the framework accuracy is given by:

$$
\begin{aligned}
\delta \mathbf{e}_{\text {nav }}(t) & =\left\|\tilde{\mathbf{y}}_{d}(t)-\mathbf{y}_{d}(t)\right\| \\
\delta \mathbf{e}_{\text {gui }}(t) & =\left\|\left(\tilde{\mathbf{y}}_{d}(t)-\tilde{\mathbf{y}}_{c}(t)\right)-\left(\mathbf{y}_{d}(t)-\mathbf{y}_{c}(t)\right)\right\|
\end{aligned}
$$

where all quantities are expressed in the same reference frame, as for example the TOD. In the navigation case, as the chief state is known from the onboard absolute navigation system, it is important to minimize the two-way error of the mean/osculating elements conversions (i.e., $\mathrm{T}_{2} \circ \mathrm{T}_{2}^{-1}$ ), in agreement with the results in [25] where the effect of using different transformations was compared. In the guidance set-up, the error in the relative state is decoupled from the one in the absolute state by subtracting $\tilde{\mathbf{y}}_{c}(t)$. As a matter of fact, in the majority of formation-flying applications the payload poses requirements on the relative control and not, simultaneously, on the absolute orbit. For remote sensing applications, the payload measurements are processed on-ground on the basis of post-facto precise orbit determination products [45]. In this way, the computation of $\overline{\boldsymbol{\alpha}}_{c 0}$ is not required to the same precision of that for semi-analytical long-term orbit propagation. Moreover, the propagation of $\overline{\boldsymbol{\alpha}}_{c}$ at later time can be based on a satellite theory second-order only for the dominant $J_{2}$ contribution, still providing accurate enough performance with simple and compact algorithms.

In a pure simulation environment (e.g., when no flight-data are available) the reference quantities $\mathbf{y}_{d}(t)$ and $\mathbf{y}_{c}(t)$ are numerically propagated (using the proper force field) starting from some known initial conditions. Note that in the scheme flow of Fig. 1 the $\mathbf{y}_{d 0}^{\mathrm{EME}}$ is not given. As for the numerical 
assessment of section $\mathrm{V}$, such initial condition is computed according to the chart in Fig. 2, to allow the definition of the relative state at initial time directly in mean ROEs. Note that in Figures 1 and 2, the symbol + denotes the computation of the deputy's $\boldsymbol{\alpha}_{d}$ from chief's orbital elements and the ROEs using the definition of Eq. (1).

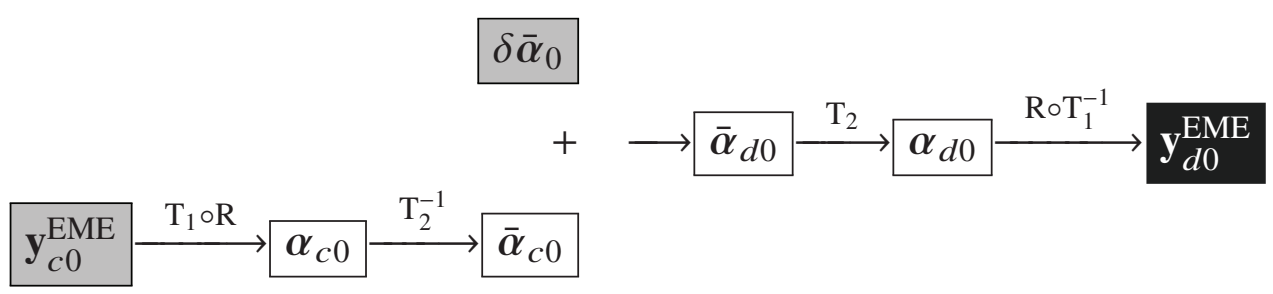

Fig. 2 Deputy initial conditions for the reference orbit.

\section{Osculating/Mean Orbital Elements Conversions}

This section describes the algorithm implemented in the elements transformations, where $\mathrm{T}_{2}$ is the direct conversion from mean to osculating elements. On the contrary, the extraction of the mean elements from the osculating set is referred as to the inverse transformation $\mathrm{T}_{2}^{-1}$.

\section{A. Hamiltonian approach for the main problem}

The analytical solution of the $J_{2}$ problem is computed using Hamiltonian perturbation theory by Lie transforms [46]. In particular, three consecutive canonical transformations are applied to obtain the secular terms of the solution up to the third order of $J_{2}$, whereas the periodic terms are computed up to the second order of $J_{2}$. Thus, the elimination of the parallax [47-49] is applied first to remove non-essential short-period effects from the original Hamiltonian. This pre-processing casts the Hamiltonian into a suitable form that eases the complete removal of the mean anomaly up to higher orders in a following Delaunay normalization [50]. A final Lie transformation eliminates the remaining long-period terms, which are related to the argument of the perigee, yielding a completely reduced, integrable Hamiltonian from which the secular frequencies are easily derived. The analytical solution constructed in this way is free from singularities related to equatorial or circular orbits, yet it cannot cope with the critical inclination resonance. All the transformations 
have been computed in closed form of the eccentricity. However, in view of the solution is only applied to LEO orbits, for better efficiency in the evaluation the periodic corrections have been expanded in powers of the eccentricity. Thus, the first-order corrections are accurate to the order of $J_{2} e^{3}$, whereas the second-order corrections are accurate to the order of $J_{2}^{2} e$. As a result, the mean-to-osculating transformations are written as follows:

$$
\left\{\begin{array}{l}
\widehat{\boldsymbol{\alpha}}=\overline{\boldsymbol{\alpha}}+f_{\text {perigee }}^{(1)}(\overline{\boldsymbol{\alpha}})+\frac{1}{2} f_{\text {perigee }}^{(2)}(\overline{\boldsymbol{\alpha}}) \\
\widetilde{\boldsymbol{\alpha}}=\widehat{\boldsymbol{\alpha}}+f_{\text {delaunay }}^{(1)}(\widehat{\boldsymbol{\alpha}})+\frac{1}{2} f_{\text {delaunay }}^{(2)}(\widehat{\boldsymbol{\alpha}}) \\
\boldsymbol{\alpha}=\widetilde{\boldsymbol{\alpha}}+f_{\text {parallax }}^{(1)}(\widetilde{\boldsymbol{\alpha}})+\frac{1}{2} f_{\text {parallax }}^{(2)}(\widetilde{\boldsymbol{\alpha}})
\end{array}\right.
$$

starting from the mean set $\overline{\boldsymbol{\alpha}}$ to obtain the osculating set $\boldsymbol{\alpha}$ in three steps, with $f_{\bullet}^{(1)}$ and $f_{\bullet}^{(2)}$ denoting respectively the first-order and second-order corrections. The functional expressions for the perigee, delaunay, and parallax transformations are reported in the supplemental appendix. The inverse transformation, instead, is given by:

$$
\left\{\begin{array}{l}
\widetilde{\boldsymbol{\alpha}}=\boldsymbol{\alpha}-f_{\text {parallax }}^{(1)}\left(\boldsymbol{\alpha}^{\mathrm{osc}}\right)+\frac{1}{2} g_{\text {parallax }}^{(2)}\left(\boldsymbol{\alpha}^{\mathrm{osc}}\right) \\
\widehat{\boldsymbol{\alpha}}=\widetilde{\boldsymbol{\alpha}}-f_{\text {delaunay }}^{(1)}(\widetilde{\boldsymbol{\alpha}})+\frac{1}{2} g_{\text {delaunay }}^{(2)}(\widetilde{\boldsymbol{\alpha}}) \\
\overline{\boldsymbol{\alpha}}=\widehat{\boldsymbol{\alpha}}-f_{\text {perigee }}^{(1)}(\widehat{\boldsymbol{\alpha}})+\frac{1}{2} g_{\text {perigee }}^{(2)}(\widehat{\boldsymbol{\alpha}})
\end{array}\right.
$$

to compute $\overline{\boldsymbol{\alpha}}$ from $\boldsymbol{\alpha}$. The second-order inverse corrections $g_{\bullet}^{(2)}$ are also reported in the supplemental appendix.

\section{Accuracy of the analytical transformation}

This section presents the accuracy analysis of the previously developed transformation and compares it with different other approaches from the literature. In particular the following methodologies are considered. The first-order mapping described in Appendix F of Ref. [51] (hereafter labeled as SJ-1st, from the initials of the names of the authors) and used in the flight software of the AVANTI experiment [3]. Such method is based on Brouwer's satellite theory [52] with Lyddane's modification [53] to accommodate small eccentricity and small inclination orbits. Due to the first order truncation, direct and inverse transformations simply differ by a sign. The first-order transformation developed in Ref. [10] using the Brouwer's generating functions re-arranged to produce a 
single transformation from mean to osculating elements. In this comparison the expression given in Eq. (42) of Ref. [10], and not its Jacobian, is implemented. This approach is labeled GA-1st, again from the initials of the names of the authors of Ref. [10]. The implementation of the short- and long-periodic corrections provided by Brouwer in [52] though using Lyddane's modification, taking only into account the effects due to $J_{2}$. Finally, the implementation of the short- and long-periodic corrections provided by Eckstein and Hechler in [37], which presents a second-order satellite theory suitable for near-circular orbits (eccentricity of the order of the $J_{2}$ ) that improves the one originally developed by Ustinov in [54]. Although Ref. [37] is developed to account for zonal terms up to order- 6 and tesseral terms up to order- 4 degree- 4 , here only the corrections related to the $J_{2}$ are considered. In particular, short-periodic corrections include terms of $J_{2}, J_{2} e$, and $J_{2}^{2}$ (this latter only for the semi-major axis); whereas the long-periodic ones include terms of $J_{2}, J_{2} e, J_{2} e^{2}, J_{2}^{2}$, and $J_{2}^{2} e$.

Note that in the last two cases the inverse transformation is not known in explicit form. Therefore, the osculating-to-mean conversion is computed iteratively with a fixed-point numerical scheme $[55,56]$, and the methodologies are respectively labeled B-I and E-I (taking the authors' initials and "I" standing for iterative). The numerical loop is initialized with the osculating set, and, to ease the convergence, the semi-major axis component of the non-singular elements' set is normalized using the known osculating value. In this way the threshold determining the terminating condition can be set to $1 \mathrm{e}-8$ and it is generally satisfied within three iterations [25].

In order to compare the performances of all the approaches mentioned so far, a reference scenario of a near-circular (i.e., $e=0.001$ ) orbit at $500 \mathrm{~km}$ of height is considered. This orbit is propagated through numerical integration (Dormand and Prince DO-PRI8, fixed time step of $10 \mathrm{~s}$ ) of the equations of motion subject to $J_{2}$ only. At each step, the inertial state, in the TOD reference system, is transformed into osculating elements, which then represent the true set of orbital elements.

Figure 3 shows the mean elements, resulting from the inverse transformation at each integration step; the analytical transformation developed in Section III A is labeled A-2nd, where "A" stands for analytical. One can note that the obtained mean values vary over the one orbit period time as result of the transformation error and of the matching imposed by the iterative process (for B-I and 

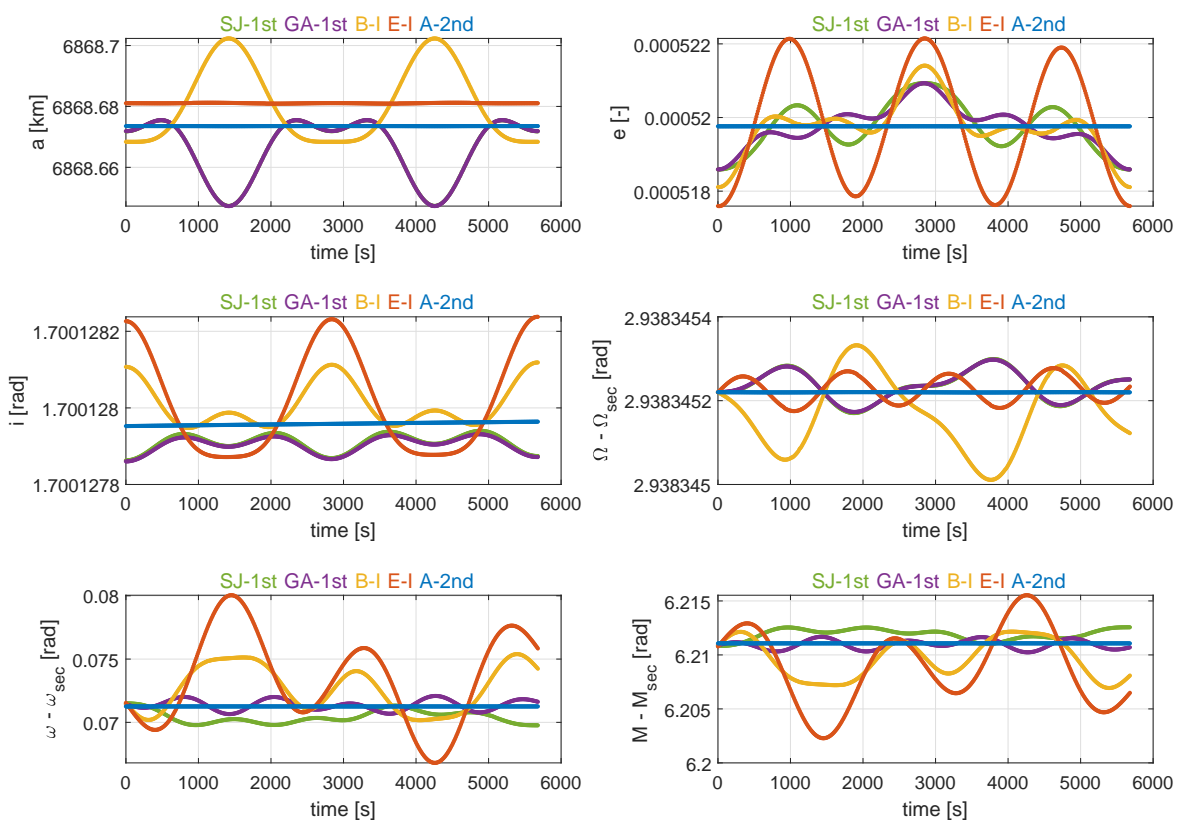

Fig. 3 Mean elements over time (1 orbit) obtained from the inverse transformation.

E-I only). The fact that the conversion error depends on when it is carried out is a relevant aspect for those spaceborne guidance algorithms executed at times corresponding to different mean anomalies of the orbit. In addition, the size of the error depends on the accuracy of the transformation method, with the two second-order approaches (i.e., E-I and A-2nd) achieving better results. For the anomalies, the plot shows the values to the net of the secular effect, which is obtained through linear fitting of the corresponding data. The focus of formation flying is on the relative motion and thus on the difference of the transformation error between the chief and the deputy orbits. Clearly, part of the absolute conversion error cancels because the orbits are similar and the satellites close. Nevertheless, the bigger the oscillation in mean-value the larger the relative error may be. The variation of mean-values for all the components except for the semi-major axis is indeed negligible for propagation time spans of few days (see Fig. 3). An error in the relative semi-major axis, instead, turns into an error in the secular component of the relative motion (a kind of an artificial drift) degrading remarkably the overall accuracy of the solution propagated over time.

Figure 4 shows the semi-major axis component only (i.e., the one responsible for the change in phasing between the chief and the target), showing the evolution of the mean value with respect to 


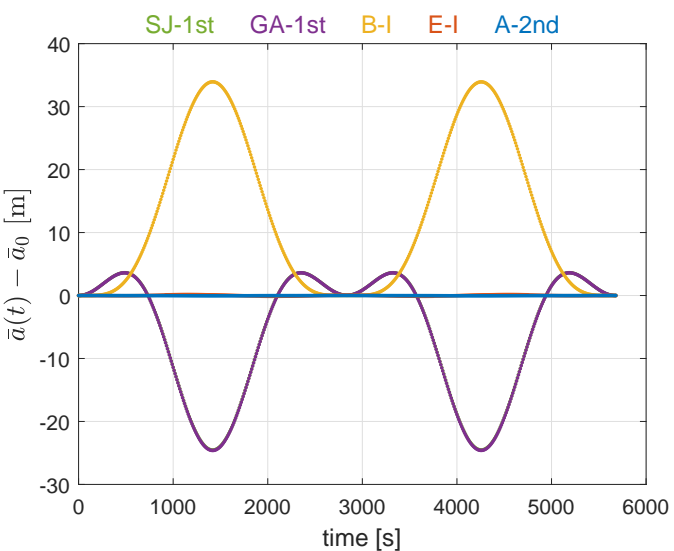

a) All considered methods.

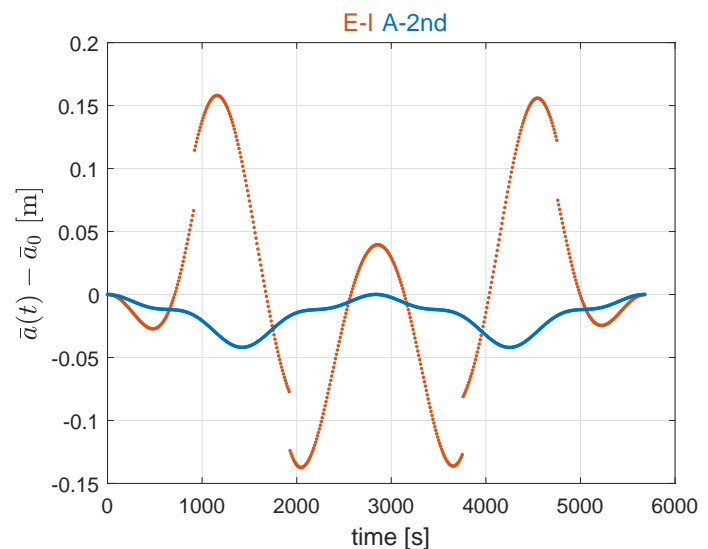

b) Only second-order methods.

Fig. 4 Semi-major axis component; plotting its value minus mean value at initial time.

the value obtained at the first step $\bar{a}\left(t_{0}\right)=\bar{a}_{0}$. In the left view all the approaches are depicted; note that SJ-1st and GA-1st are equivalent. Whereas in the right one the focus is on the second-order methods. This allows comparing their performances and visualizing the effects introduced by the simplification of terms performed in E-I, where the second order $J_{2}$-solution is not fully consistent as $J_{2}^{2}$ terms were neglected everywhere except for in the semi-major axis [36, 37]. Note that the discontinuities in the results of E-I are due to the numerical process, which is repeated at each considered time step.

\section{B. The complete transformations}

The A-2nd transformation is used to cancel the effect of the $J_{2}$, providing better accuracy than E-I while avoiding a numerical iterative process. The algorithm is then completed by applying the periodic corrections due to any remaining element of the zonal, sectorial, and tesseral harmonics based on the Kaula's first-order method. This formulation, in fact, is very convenient for the recursive implementation of the higher order and degree components of the geopotential. The use of a first-order theory for the terms of order greater than two is reasonable, as they are of order of $J_{2}^{2}$.

As explained in the introduction, in formation flying applications the highest accuracy is actually needed only for the semi-major axis component (i.e., the one responsible for the secular growth of 
the relative orbit). Accordingly, to fulfill the most compact algorithm, the Kaula-based corrections are only performed for the semi-major axis, as follows [35]:

$$
\begin{aligned}
& \Delta a_{l m p q}= \frac{\mu_{e} R_{e}^{l}}{n a^{l+2}} 2 F_{l m p}(i) G_{l p q}(e)(l-2 p+q) \frac{S_{l m p q}}{\dot{\Psi}_{l m p q}} \\
& S_{l m p q}= \begin{cases}+C_{l m} \cos \Psi_{l m p q}+S_{l m} \sin \Psi_{l m p q} & \text { if } l-m \text { is even } \\
-S_{l m} \cos \Psi_{l m p q}+C_{l m} \sin \Psi_{l m p q} & \text { if } l-m \text { is odd }\end{cases} \\
& \Psi=(l-2 p) \omega+(l-2 p+q) M+m(\Omega-\Theta)
\end{aligned}
$$

where $\Delta a$ is the change in semi-major axis, $l$ and $m$ are order and degree of the geopotential with coefficients $C_{l m}$ and $S_{l m}, p \leq l$ is an index, $F_{l m p}$ are the inclination functions, $G_{l p q}$ are the eccentricity functions (the short periodic ones are series, but for small eccentricities is enough to consider the index $q \in[-2,2]$ [57]), and $\Theta$ is the Greenwich sideral time.

The transformations obtained combining Hamiltonian and Kaula's approaches are labeled KA$\mathbf{l} \times \mathbf{m}$, being $l$ and $m$ respectively order and degree of the geopotential terms accounted in the corrections. No problems of singularity arise in the Kaula-phase, since this is used only to correct the semi-major axis (e.g., there are no issues due to the $e$ at denominator of the $\Delta e$ part of the eccentricity vector correction, for the cases in which it does not simplify with the $e$ coming from the eccentricity functions). Moreover, the correction of the semi-major axis requires only the computation of inclination, short-, and long-periodic eccentricity functions but none of their derivatives with respect to their arguments. Finally, the first order correction is the same in both directions, except for the sign. The effect of using the $\mathbf{K A -} \times \mathbf{l} \times \mathbf{m}$ algorithm on the overall numerical accuracy achievable in the modeling of the relative motion is presented later in Section V.

\section{Relative motion model in mean elements}

After having applied the transformations $\mathrm{T}_{2}^{-1}$, the only mean elements that present a secular variation are $\Omega, \omega$, and $M$, generated by even zonal harmonics only. At this point ROE-based models in literature derive the plant matrix of the linearized relative dynamics by expanding to the first order the time derivatives of of the chief satellite $\dot{\overline{\boldsymbol{\alpha}}}_{c}[18,19]$. Following the approach of 
$[10,15,28]$, instead, here the mean orbit of the chief $\overline{\boldsymbol{\alpha}}_{c}(t)$

$$
\begin{aligned}
& e_{x}(t)=e_{x 0} \cos \left(\dot{\omega}\left(t-t_{0}\right)\right)-e_{y 0} \sin \left(\dot{\omega}\left(t-t_{0}\right)\right) \\
& e_{y}(t)=e_{x 0} \sin \left(\dot{\omega}\left(t-t_{0}\right)\right)+e_{y 0} \cos \left(\dot{\omega}\left(t-t_{0}\right)\right) \\
& \Omega(t)=\dot{\Omega}\left(t-t_{0}\right)+\Omega_{0} \\
& u(t)=\dot{\omega}\left(t-t_{0}\right)+\dot{M}\left(t-t_{0}\right)+u_{0}
\end{aligned}
$$

is expanded ( $a$ and $i$ are constants). In Eq. (6) the ${ }^{-}$notation and the "c" subscript are dropped dealing with mean quantities of the chief only. In this way, the STM is directly derived, with no need to integrate the linearized system of equations. Moreover, the extension to further terms of the Taylor expansion, as needed to model rendezvous or larger formations, becomes straightforward.

\section{A. The first-order state-transition matrix}

The STM is obtained by retaining the first-order only expansion with respect to $a, e_{x}, e_{y}$, and $i$ rearranged so that $\delta \boldsymbol{\alpha}(t)=\Phi\left(\boldsymbol{\alpha}_{0}, \Delta t\right) \delta \boldsymbol{\alpha}_{0}$ with the ROEs defined in Eq. (1):

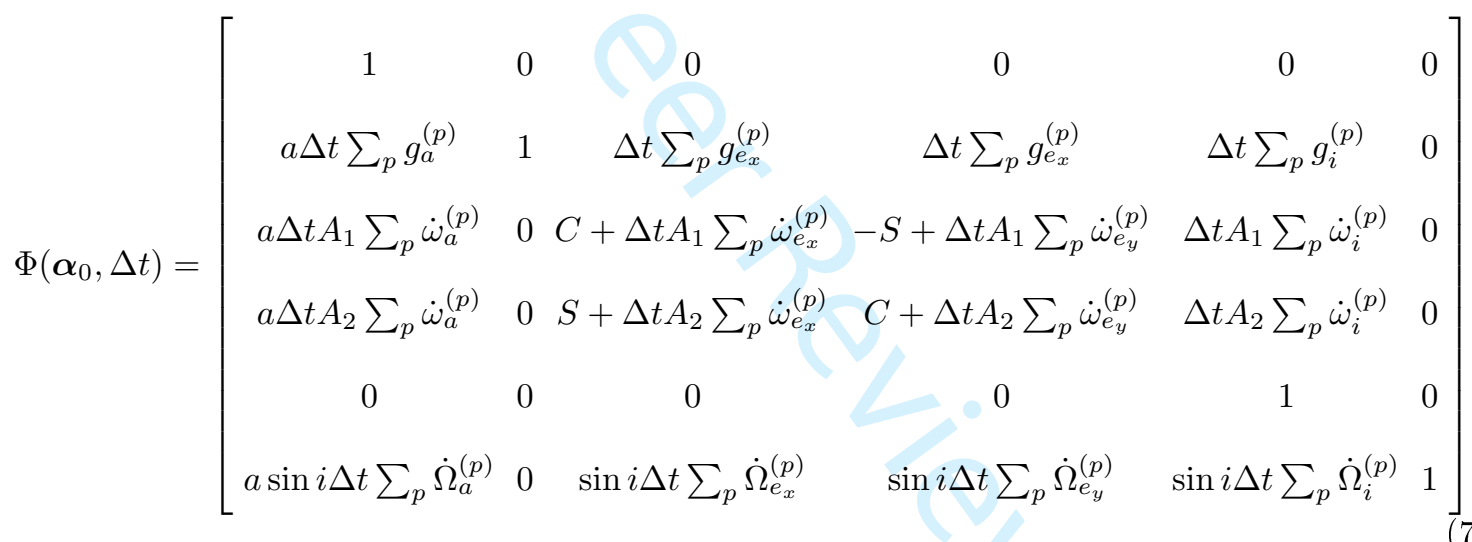

where $\Delta t=t-t_{0}$ is the time elapsed from the starting epoch, $p$ is the index of the ordered set $\mathrm{J}:\left\{J_{0}, J_{2}, J_{2}^{2}, J_{4}, J_{6}\right\}$ collecting the considered zonal contributions, and the subscript notation $f_{x}^{(p)}$ is used to denote the partial derivatives of $f^{(p)}$ - generated by the $\mathrm{J}\{p\}$ contribution - with respect to the element $x$. The notation $J_{0}$ has been used to express the Keplerian solution, for which only $M$ varies over time with $\dot{M}=n$, being $n$ the unperturbed mean motion. The remaining functions 
that appear in Eq. (7) are:

$$
\begin{aligned}
& S=\sin \left(\sum_{p} \dot{\omega}^{(p)} \Delta t\right) \quad C=\cos \left(\sum_{p} \dot{\omega}^{(p)} \Delta t\right) \\
& A_{1}=-\left(S e_{x 0}+C e_{y 0}\right) \quad A_{2}=+\left(C e_{x 0}-S e_{y 0}\right) \\
& g=\dot{\omega}+\dot{M}+\dot{\Omega} \cos i
\end{aligned}
$$

where $\dot{\omega}$ and $\dot{\Omega}$ respectively are the time derivatives of the argument of perigee and of the longitude of ascending node. The STM structure of Eq. (7) accounts for whatever order of the geopotential (i.e., with $\mathrm{J}$ up to the consistent $p$ term and $\mathrm{T}_{2}^{-1}$ of proper order and degree), and is valid for whatever eccentricity without introducing any further approximation. Thus it generalizes the ones of [18, 19], with $\Phi$ supporting a straightforward geometrical representation of the evolution of the mean ROEs over time. Given its relevance in the design of formation keeping and reconfiguration algorithms, the functional expression of Eq. (7) including only the dominant $J_{2}$ effect to the first-order is provided in the supplemental appendix. The description of the qualitative evolution of the ROEs in their space has been discussed in several works $[17-19,39,40]$. To assess the relevance of the effects due to higher terms of the geopotential one can refer to the structures of the partial derivatives presented in Table 1. There, $K_{2}=J_{2}\left(R_{\oplus} / a\right)^{2}, K_{22}=K_{2}^{2}, K_{4}=J_{4}\left(R_{\oplus} / a\right)^{4}, K_{6}=J_{6}\left(R_{\oplus} / a\right)^{6}, R_{\oplus}$

\begin{tabular}{|c|c|c|c|c|c|c|}
\hline & $(\dot{\Omega} \text { or } \dot{\omega})_{a}$ & $(\dot{\Omega} \text { or } \dot{\omega})_{e_{*}}$ & $(\dot{\Omega} \text { or } \dot{\omega})_{i}$ & $\dot{M}_{a}$ & $\dot{M}_{e_{*}}$ & $\dot{M}_{i}$ \\
\hline$J_{2}$ & $c K_{2} \frac{n}{\eta^{4}} \frac{1}{a} f(i)$ & $c K_{2} \frac{n}{\eta^{6}} e_{*} f(i)$ & $c K_{2} \frac{n}{\eta^{4}} f(i)$ & $c K_{2} \frac{n}{\eta^{3}} \frac{1}{a} f(i)$ & $c K_{2} \frac{n}{\eta^{5}} e_{*} f(i)$ & $c K_{2} \frac{n}{\eta^{3}} f(i)$ \\
\hline$J_{2}^{2}$ & $c K_{22} \frac{n}{\eta^{8}} \frac{1}{a} \hat{f}$ & $c K_{22} \frac{n}{\eta^{10}} e_{*} \hat{f}$ & $c K_{22} \frac{n}{\eta^{8}} \hat{f}$ & $c K_{22} \frac{n}{\eta^{9}} \frac{1}{a} \hat{f}$ & $c K_{22} \frac{n}{\eta^{11}} e_{*} \hat{f}$ & $c K_{22} \frac{n}{\eta^{9}} \hat{f}$ \\
\hline$J_{4}$ & $c K_{4} \frac{n}{\eta^{8}} \frac{1}{a} \hat{f}$ & $c K_{4} \frac{n}{\eta^{10}} e_{*} \hat{f}$ & $c K_{4} \frac{n}{\eta^{8}} \hat{f}$ & $c K_{4} \frac{n}{\eta^{7}} \frac{1}{a} \hat{f}$ & $c K_{4} \frac{n}{\eta^{9}} e_{*} \hat{f}$ & $c K_{4} \frac{n}{\eta^{7}} \hat{f}$ \\
\hline$J_{6}$ & $c K_{6} \frac{n}{\eta^{12}} \frac{1}{a} \hat{f}$ & $c K_{6} \frac{n}{\eta^{14}} e_{*} \hat{f}$ & $c K_{6} \frac{n}{\eta^{12}} \hat{f}$ & $c K_{6} \frac{n}{\eta^{11}} \frac{1}{a} \hat{f}$ & $c K_{6} \frac{n}{\eta^{13}} e_{*} \hat{f}$ & $c K_{6} \frac{n}{\eta^{11}} \hat{f}$ \\
\hline
\end{tabular}
is the Earth radius and $\eta=\sqrt{\left(1-e_{x}^{2}-e_{y}^{2}\right)}$.

Table 1 Structure of the partial derivatives of $\dot{\Omega}, \dot{\omega}$, and $\dot{M}$ with respect to $a, e_{x}, e_{y}$, and $i$.

Note: $c$ numerical coefficients, $\hat{f}=f\left(\cos ^{2} i, e^{2}\right)$ different for each entry, and $e_{*}$ denotes either $e_{x}$ or $e_{y}$. 


\section{B. The second-order state-transition tensor}

When performing active debris removal missions, the chaser satellite undergoes phases of rendezvous from far-range and phases in which its relative orbit with respect to the target's one might be quite large (e.g., after the coarse orbit phasing when the far-range relative sensors start detecting the target object). In these cases a first-order expansion in the orbital elements might not be enough to preserve the accuracy of the relative motion model and/or to support relative initial orbit determination or relative navigation algorithms. With the selected parametrization, the inclusion of further terms of expansion of the unperturbed dynamics is trivial, being $g$ (and thus $\delta \lambda$ ) only function of the mean semi-major axis. The second-order correction due to the $p$ term of the $\mathrm{J}$ set can be included considering the $\mathrm{H}$ matrix associated to a function $z$ (expressed by the notation $\mathrm{H}_{z}$ ), which assembles as follows the double partials with respect the orbital elements:

$$
\mathrm{H}_{z}=\left[\begin{array}{cccccc}
a^{2} z_{a, a} & 0 & a z_{a, e_{x}} & a z_{a, e_{y}} & a z_{a, i} & 0 \\
0 & 0 & 0 & 0 & 0 & 0 \\
a z_{a, e_{x}} & 0 & z_{e_{x}, e_{x}} & z_{e_{x}, e_{y}} & z_{e_{x}, i} & 0 \\
a z_{a, e_{y}} & 0 & z_{e_{x}, e_{y}} & z_{e_{y}, e_{y}} & z_{e_{y}, i} & 0 \\
a z_{a, i} & 0 & z_{e_{x}, i} & z_{e_{y}, i} & z_{i, i} & 0 \\
0 & 0 & 0 & 0 & 0 & 0
\end{array}\right]
$$

being $z$ on turn $g^{(p)}, \dot{\Omega}^{(p)}, e_{x}^{(p)}$, and $e_{y}^{(p)}$. Here $z_{a, e_{x}}$, for example, is the short notation for $\frac{\partial^{2} z}{\partial a \partial e_{x}}$. Thus, the unperturbed part associated to $J_{0}$ simply reduces to $\dot{M}_{a, a}$ in the first component of $\mathrm{H}_{g^{(0)}}$. For the eccentricity vector components, the partial derivatives $e_{* j, k}$ are computed from Eq. (6). As a result, the relative motion model in the mean $\mathrm{ROE}$ space can be written to include the second-order correction

$$
\begin{aligned}
& \delta \boldsymbol{\alpha}(t)=\Phi\left(\boldsymbol{\alpha}_{0}, \Delta t\right) \delta \boldsymbol{\alpha}_{0}+\frac{1}{2} \Psi\left(\boldsymbol{\alpha}_{0}, \Delta t\right) \otimes \delta \boldsymbol{\alpha}_{0} \otimes \delta \boldsymbol{\alpha}_{0} \\
& \Psi\left(\boldsymbol{\alpha}_{0}, \Delta t\right)=\left[\begin{array}{llllll}
\mathrm{O}_{6 \times 6} & \Delta t \mathrm{H}_{g} & \mathrm{H}_{e_{x}} & \mathrm{H}_{e_{y}} & \mathrm{O}_{6 \times 6} & \sin i \Delta t \mathrm{H}_{\dot{\Omega}}
\end{array}\right]
\end{aligned}
$$

where $\Psi$ is the second-order state-transition tensor of dimension $6 \times 6 \times 6$ built from the $\mathrm{H}_{z}$ as prescribed in Eq. (10) and $\otimes$ is the dyadic product. For the sake of readability the notation.$^{(p)}$ has been dropped. Nevertheless, when accounting for only the unperturbed and $J_{2}$ (to the firstorder) terms, the $\Psi$ in Eq. (10) is the ROE-based version of the STT of Yang et al. [15]. Again 
by using ROEs of Eq. (1) for the parametrization, the tensor presents a sparser structure and the generalization to include further terms becomes straightforward. Note that in [15] in order to link $\Delta u$ to the variation of true argument of latitude, a Taylor expansion of the eccentric argument of latitude is needed, which requires further hypotheses on the size of the orbital elements' differences, and thus restricts the range of applicability of such second-order model.

Figures 5 and 6 show the sizes of the corrections due higher zonal terms to the first order and second-order expansions of Keplerian and $J_{2}$ terms. There, the mean elements of the chief are $a=710+R_{\oplus} \mathrm{km}, e=0.001$, and $i=98.17^{\circ}$ (i.e., a sun-synchronous condition). In Fig. 5 the size of the relative orbit is considered. Therefore, the same case of Ref. [8] is taken, where the relative orbit is parametrized as follows: bounded $\delta a=0$, centered, $\delta \lambda=0$, (anti-)parallel configuration with no $y$ component $\delta e_{y}=\delta i_{y}=0$, and same magnitude of the relative eccentricity and inclination vectors $\delta e=\delta i$. Accordingly, this case presents a worst-case scenario for the effects produced by the non-spherically symmetric mass distribution, and the orbit size can be parametrized in the nondimensional maximum separation between the spacecraft $\delta r_{\max } / a_{c}$ (as done in [8]). In this case, the second-order expansion due to $J_{2}$ becomes more relevant for orbits larger than $\delta r_{\max } / a_{c}>1.45 \cdot 10^{-3}$ (i.e., $a \delta e \approx 4600 \mathrm{~m}$ ) for the relative mean longitude and $\delta r_{\max } / a_{c}>2.8 \cdot 10^{-3}$ (i.e., $a \delta e \approx 8800 \mathrm{~m}$ ) for the relative eccentricity vector.

The plots of Fig. 6 regard the case of a passively safe drifting orbit, as typically used for noncooperative rendezvous. Accordingly, $a \delta a$ in meters vary to parametrize the magnitude of the drift in flight direction, $\delta e_{x}=\delta i_{x}=0$, and the magnitude of relative eccentricity and inclination vectors are moderate (the orbit size has been already shrink, to fit better in the field of view of the relative navigation sensors). Given such limited size of the orbit, the corrections in $\delta \lambda$ are much more relevant. Moreover, here the second-order expansion of the unperturbed term becomes more relevant for drifts larger than $a \delta a>135 \mathrm{~m}$, a common value for far-range rendezvous looking at the flight-data of ARGON (Fig. 13 of Ref. [18]) and AVANTI (Fig. 26 of Ref. [6]). 

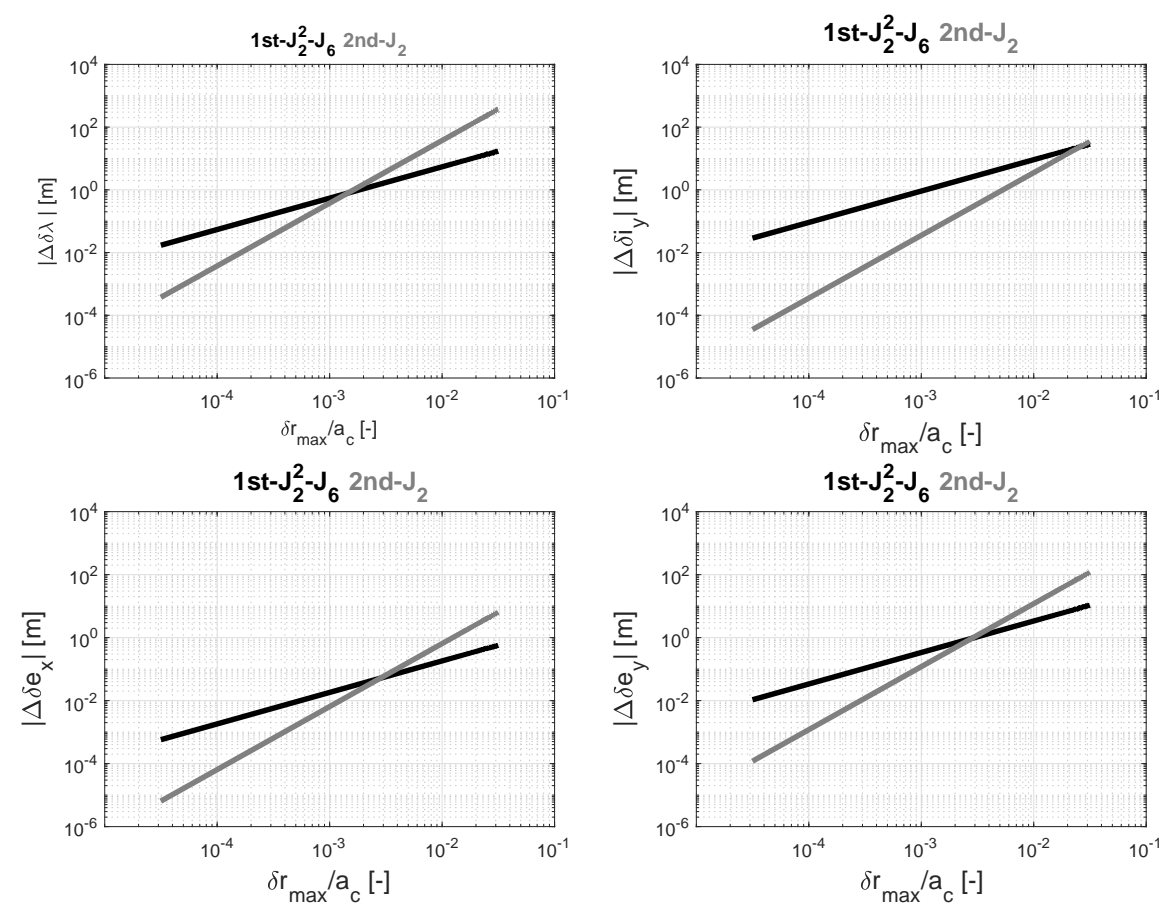

Fig. 5 ROE corrections for relative orbits of increasing size $\delta \boldsymbol{\alpha}_{0}=(0,0, k, 0, k, 0)^{\mathbf{T}}$.
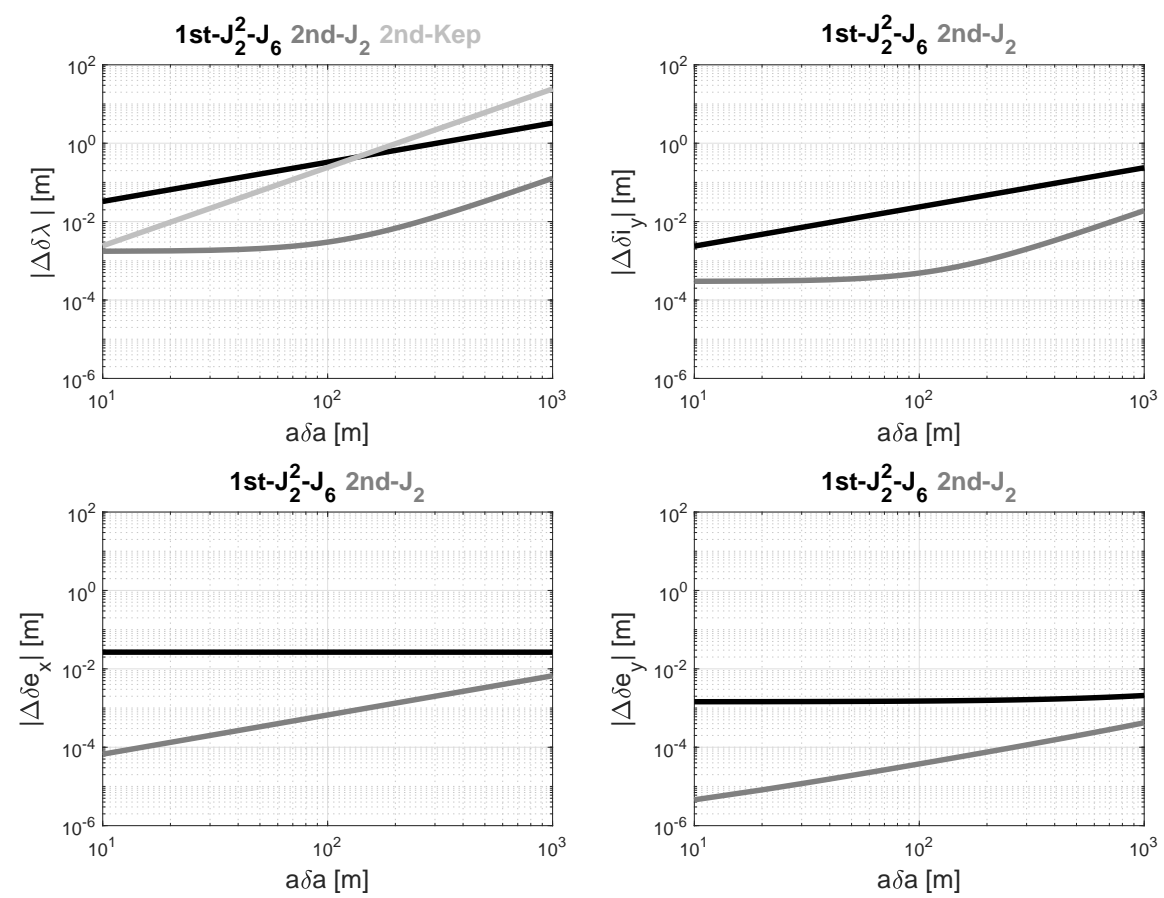

Fig. 6 ROE corrections for drifting passively safe relative orbit $a \delta \boldsymbol{\alpha}_{0}=(k, 4500,0,250,0,300)^{\mathbf{T}} \mathbf{m}$. 


\section{Results}

This section presents the numerical accuracy that can be achieved by adopting the proposed framework. To this end, the reference states of the deputy and chief satellites $\mathbf{y}_{\text {TOD }}$ are obtained by applying the trasformation $\mathrm{R}$ to the ones out of the numerical integration carried out in EME, starting from the initial conditions computed according to Fig. 2, assuming a force field specified case-by-case.

The presented analysis is carried out assuming a scenario from the PRISMA (Prototype Research Instruments and Space Mission Technology Advancement) mission [58]. Accordingly, the orbit of the chief is taken as sun-synchronous, dusk-dawn, near-circular $e=0.001$ orbit, at the same height used in section IV B (i.e., $710 \mathrm{~km}$ ). Moreover, for the inclusion of the effects of the non-conservative perturbations, the deputy and chief satellites are customized on the Tango and Mango spacecraft respectively, whose properties are recalled in Table 2 of Ref. [8]. The PRISMA scenario has been chosen as representative of LEO orbits with a height where the effect of the differential aerodynamic drag is weak (see section 7 of Ref. [18]). Beyond pure formation flying applications, for example devoted to remote sensing, this orbital area is particularly interesting for future active debris removal activities.

\section{A. The effect of the initial conditions}

The errors in relative position and velocity at time $t$ in the TOD frame given by Eq. (2) are weighted as:

$$
\delta \hat{\mathbf{e}}=\mathrm{W} \delta \mathbf{e}, \quad \mathrm{W}=\mathrm{I}_{6 \times 6} \cdot\left(1,1,1,1 / n_{c 0}, 1 / n_{c 0}, 1 / n_{c 0}\right)^{\mathrm{T}}
$$

using the chief's mean motion, to provide an error in dimension of length [8]. In order to assess the accuracy performance the index of merit $\nu$ over the fixed time duration $\Delta t$ is defined as:

$$
\nu_{\Delta t}=\nu(\Delta t)=\max _{t_{0, i} \in\left[t_{0}, t_{0}+\mathrm{T}_{\mathrm{ref}}\right]}\left\|\delta \hat{\mathbf{e}}\left(\Delta t, t_{0, i}\right)\right\|_{2}=\max _{i} \nu_{i}(\Delta t)
$$

Accordingly, this index provides the maximum of the norm-2 of the weighted relative error in meters, over the given elapsed time $\Delta t$, considering all possible $i$-th initial times $t_{0, i}$ contained in the reference interval $\mathrm{T}_{\text {ref }}$ (typically equal to one orbital period). The index of Eq. (12) is inspired 
from the one defined in Eq. (8) of Sullivan et al. [8] and used to compare the several relative motion models surveyed there. Though, the adopted index extends the one of [8], by considering the effect of the initial conditions, introduced by the osculating/mean elements' conversion errors. In the comparative work of Alfriend and Yan [59], instead, for each considered relative orbit, a set of 100 equally spaced initial conditions were propagated. As a result, the metric of Eq. (12) merges two fundamental aspects, namely: the quantitative result of a relative error expressed in meters (beneficial to size GNC hardware and algorithms), and the conservative principle of the worst-case condition (beneficial to provide a realistic assessment of the performance).

Regarding the relative motion, the relative orbit of the family used in Fig. 6 with $\delta a=0$ is taken and propagated with the model of Eq. (10). Note that in this condition the effects due to the higher zonal terms and due to second-order expansion are very small, and thus this model provides de-facto the same propagation of the STM used to develop the spaceborne GNC system of AVANTI (i.e., Eq. (6) of Ref. [3]). Here, in fact, the focus is on assessing the benefits of using the $\mathrm{T}_{2}$ of section III in the framework. The summary of the performed simulations is provided in Table 2, where the results associated to the navigation error $\delta \mathbf{e}_{\text {nav }}$ are reported. For each performed simulation, the $\mathrm{T}_{\text {ref }}$ interval is taken as 5940 seconds that corresponds to circa the unperturbed orbital period of the chief satellite. By considering a time granularity of 1 minute for the initial times $t_{0, i}$, a population of 99 different initial conditions is employed for each run. The value of the index $\nu$ is provided for two simulation legs, corresponding to 1-day (i.e., $14.5 \mathrm{~T}_{\text {ref }}$ ) and 2-day long (i.e., $29 \mathrm{~T}_{\text {ref }}$ ) time durations.

Table 2 Summary of performed simulations. Index scores are given for the error of the navigation set-up.

\begin{tabular}{|c|c|c|c|c|c|c|c|c|c|}
\hline \multirow[t]{2}{*}{ Transf. } & \multicolumn{3}{|c|}{ Geopot. $6 \times 6$} & \multicolumn{3}{|c|}{ Geopot. $30 \times 30$} & \multicolumn{3}{|c|}{ Geopot. $30 \times 30 \&$ Others } \\
\hline & Plot & $\nu_{1 \text {-day }}, \mathrm{m}$ & $\nu_{2 \text {-day }}, \mathrm{m}$ & Plot & $\nu_{1 \text {-day }}, \mathrm{m}$ & $\nu_{2 \text {-day }}, \mathrm{m}$ & Plot & $\nu_{1 \text {-day }}, \mathrm{m}$ & $\nu_{2 \text {-day }}, \mathrm{m}$ \\
\hline SJ-1st & Fig. 7 a) & 46.47 & 61.49 & - & - & - & - & - & - \\
\hline $\mathrm{KA}-6 \times 6$ & Fig. 7 b) & 0.77 & 1.09 & Fig. 8 a) & 14.55 & 28.95 & Fig. 9 a) & 21.61 & 57.86 \\
\hline $\mathrm{KA}-10 \times 10$ & - & - & - & Fig. 8 b) & 7.67 & 14.99 & Fig. 9 b) & 14.80 & 43.63 \\
\hline
\end{tabular}

The reference orbits are generated for three different orbital dynamics. In the first two cases 


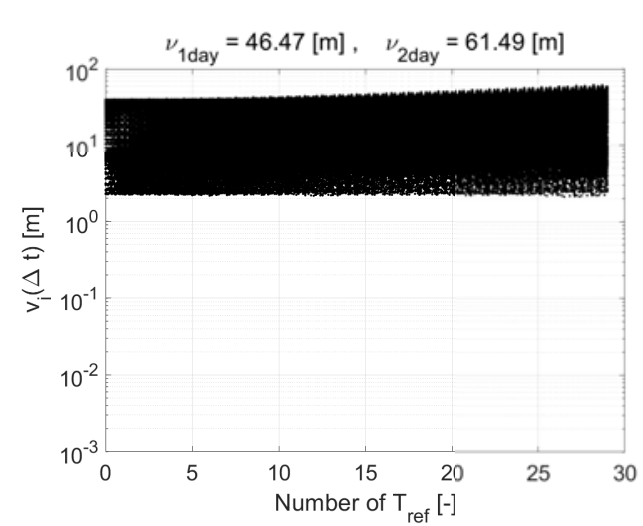

a) $T_{2}$ with SJ-1st.

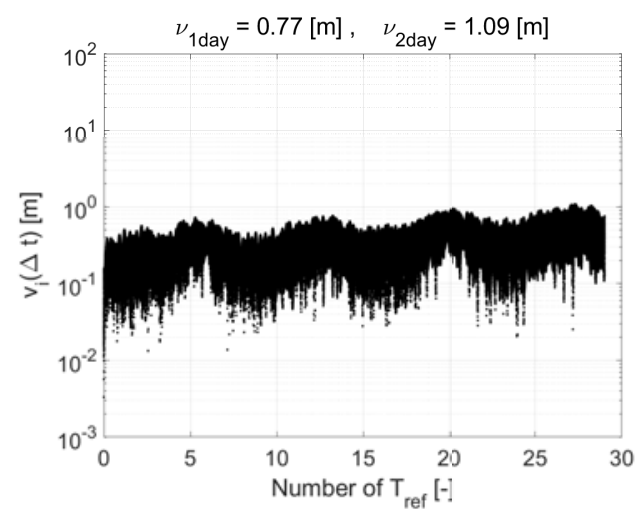

b) $\mathrm{T}_{2}$ with KA- $6 \times 6$.

Fig. 7 Accuracy of the navigation set-up: reference orbits from numerical integration in $6 \times 6$ gravity field.

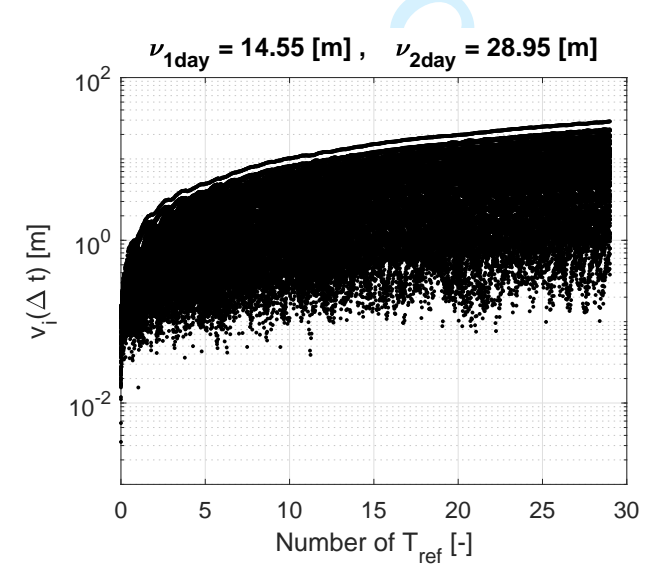

a) $\mathrm{T}_{2}$ with $\mathrm{KA}-6 \times 6$.

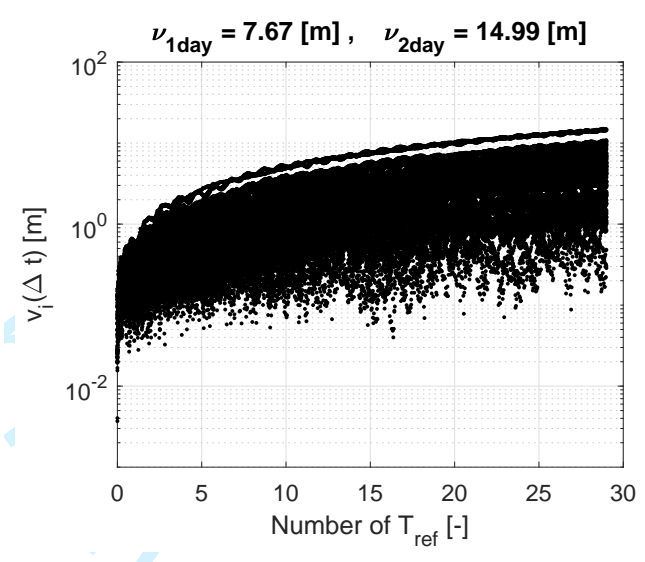

b) $\mathrm{T}_{2}$ with $\mathrm{KA}-10 \times 10$.

Fig. 8 Accuracy of the navigation set-up: reference orbits from numerical integration in $30 \times 30$ gravity field.

the non-spherically symmetric mass distribution is the solely perturbation accounted, respectively geopotential of order- 6 degree- 6 and order- 30 degree- 30 . In the last case, in addition to the $30 \times 30$ geopotential all other orbital perturbations are included (e.g., aerodynamic drag, solar radiation pressure, third-body due to Moon and Sun, tidal and relativity effects). Finally, for each performed simulation the reference to the related plot is recalled (i.e., see the columns Plot in Table 2).

Figure 7 shows the benefits of employing the osculating/mean conversion algorithm of section III against the one used in the AVANTI flight software. This comparison is performed in the case when 